04,11

\title{
Толеранс-фактор для соединений класса хантитов
}

\author{
(C) М.С. Молокеев ${ }^{1,2}$, С.О. Кузнецов ${ }^{1}$ \\ ${ }^{1}$ Сибирский фредеральный университет, \\ Красноярск, Россия \\ ${ }^{2}$ Институт ффизики им. Л.В. Киренского ФИЦ КНЦ СО РАН, \\ Красноярск, Россия \\ E-mail: msmolokeev@mail.ru
}

Поступила в Редакцию 3 июня 2020 г.

В окончательной редакции 3 июня 2020 г.

Принята к публикации 26 июня 2020 г.

Проведен анализ 85 соединений со структурой типа хантита $R M_{3}\left(\mathrm{BO}_{3}\right)_{4}$, где $R=$ редкоземельный элемент $(\mathrm{Y}, \mathrm{La}-\mathrm{Lu}), M=\mathrm{Al}, \mathrm{Sc}, \mathrm{Cr}, \mathrm{Fe}, \mathrm{Ga}$. Анализ структур позволил выявить критические смещения атомов при фазовом переходе $R 32 \leftrightarrow P 3_{1} 21$, и установить, как этими критическими смещениями можно управлять посредством вариации ионных радиусов. В итоге выведен толеранс-фактор и его пороговое значение, ниже которого структура стабильна в фазе $R 32$, а выше - в искаженной фазе $P 3{ }_{1} 21$. Формула апробирована на более 30 соединениях типа хантита и дала хорошее согласие. Поэтому ее можно с уверенностью применять для прогноза новых соединений. На данный момент толеранс-фактор позволил выявить закономерности в хантитах, которые ранее были неизвестны.

Ключевые слова: хантиты, толеранс-фактор, фазовый переход, стабильность структуры, кристаллическая структура.

DOI: $10.21883 /$ FTT.2020.11.50108.120

\section{1. Введение}

В последние десятилетия кристаллы бората представляют большой интерес благодаря широкому разнообразию структур [1]. Бораты оптически прозрачны в широком спектральном диапазоне, обладают хорошей химической и механической стабильностью. Бораты со структурой хантита (хантит - $\mathrm{CaMg}_{3}\left(\mathrm{CO}_{3}\right)_{4}$, пространственная группа $R 32$ ) представляют значительный интерес из-за ценных магнитоэлектрических $[2,3]$ и спектроскопических [4-6] свойств, перспективных для технических применений. Общая формула ханитоподобных боратов: $R M_{3}\left(\mathrm{BO}_{3}\right)_{4}$, где $R=$ редкоземельный элемент (Y, La-Lu), $M=\mathrm{Al}, \mathrm{Sc}, \mathrm{Cr}, \mathrm{Fe}, \mathrm{Ga}$.

Тип кристаллической структуры $R M_{3}\left(\mathrm{BO}_{3}\right)_{4}$ зависит от химического состава и условий кристаллизации (таблица). В каждой структуре выделяют три типа координационных полиэдров: тригональные призмы $R \mathrm{O}_{6}$, октаэдры $\mathrm{MO}_{6}$ и полиэдры $\mathrm{BO}_{3}$ в форме треугольников. В наиболее распространенной фазе с симметрией $R 32$ существует один $R \mathrm{O}_{6}$, один $M \mathrm{O}_{6}$ и два $\mathrm{BO}_{3}$ в независимой части ячейки (рис. 1). Помимо $R 32$ фазы существуют упоминания о тригональных $P 3{ }_{1} 21$, $P 321$ и моноклинных фазах $C 2 / c, C c, C 2$. Однако для хантитов до сих пор не предложена количественная мера, позволяющая оценить формирование той или иной фазы, и делать быстрый прогноз симметрии. Обычно такое мерой является толеранс-фактор - показатель стабильности и искажения кристаллических структур [7]. Первоначально он использовался только для описания структуры перовскита [8], но теперь, например, факторы толерантности также используются для ильменита [9], гранатов [10], пирохлоров [11] и халькогенидов с общей формулой $\mathrm{ABCX}_{3}[12]$.

В настоящей работе нами предложен толеранс-фактор для определения стабильности фазы $R 32$ хантитоподобных кристаллов, а также его пороговое значение, при котором происходит переход в $P 3{ }_{1} 21$-фазу. Выведенная формула может быть используема для прогноза симметрии фазы, а, следовательно, и некоторых свойств вещества, используя информацию только о предполагаемой химической формуле.

\section{2. Методика}

Для выявления закономерностей образования различных фаз хантитов было решено собрать информацию

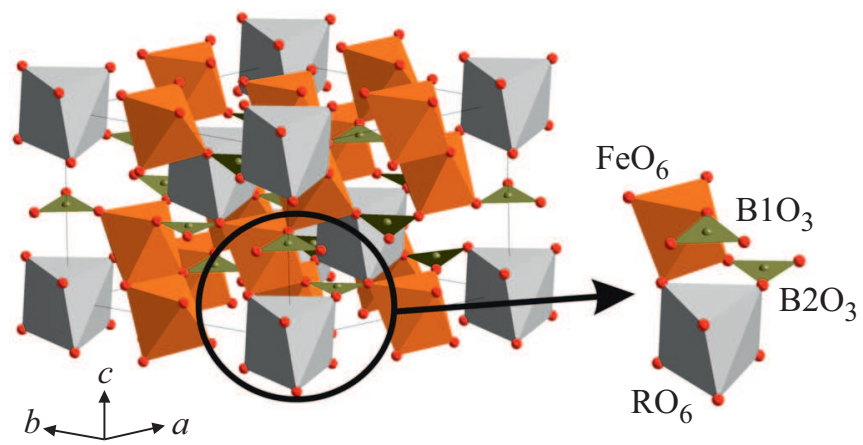

Pис. 1. Кристаллическая структура $R M_{3}\left(\mathrm{BO}_{3}\right)_{4}$ в фазе $R 32$. Независимая часть ячейки выделена кругом. 
Список основных соединений хантитов с общей химической формулой $\operatorname{LnM}\left(\mathrm{BO}_{3}\right)_{4}$ из баз данных COD и ICSD. (Copтировка соединений выполнена по увеличению толеран-фактора ( $t$-фактор) и выполнена группировка по пространственным группам)

\begin{tabular}{|c|c|c|c|c|c|}
\hline $\begin{array}{c}\text { Пространственная } \\
\text { группа }\end{array}$ & Ln & $M$ & $I R \operatorname{Ln}(\AA)$ & $\operatorname{IRMe}(\AA)$ & $t$-фактор \\
\hline$R 32$ & $\mathrm{Nd}$ & $\mathrm{Al}$ & 1.109 & 0.535 & 1.603 \\
\hline$R 32$ & $\mathrm{Eu}$ & $\mathrm{Al}$ & 1.066 & 0.535 & 1.633 \\
\hline$R 32$ & Gd & $\mathrm{Al}$ & 1.053 & 0.535 & 1.642 \\
\hline$R 32$ & $\mathrm{La}$ & $\mathrm{Fe}$ & 1.160 & 0.645 & 1.644 \\
\hline$R 32$ & $\mathrm{Nd}$ & $\mathrm{Ga}$ & 1.109 & 0.620 & 1.663 \\
\hline$R 32$ & $\mathrm{Y}$ & $\mathrm{Al}$ & 1.019 & 0.535 & 1.666 \\
\hline$R 32$ & Ho & $\mathrm{Al}$ & 1.015 & 0.535 & 1.669 \\
\hline$R 32$ & $\mathrm{Er}$ & $\mathrm{Al}$ & 1.004 & 0.535 & 1.677 \\
\hline$R 32$ & $\mathrm{Nd}$ & $\mathrm{Fe}$ & 1.109 & 0.645 & 1.680 \\
\hline$R 32$ & $\mathrm{Tm}$ & $\mathrm{Al}$ & 0.994 & 0.535 & 1.684 \\
\hline$R 32$ & $\mathrm{Eu}$ & $\mathrm{Cr}$ & 1.066 & 0.615 & 1.689 \\
\hline$R 32$ & $\mathrm{Yb}$ & $\mathrm{Al}$ & 0.985 & 0.535 & 1.690 \\
\hline$R 32$ & $\mathrm{Y}_{0.5} \mathrm{Bi}_{0.5}$ & $\mathrm{Fe}$ & 1.095 & 0.645 & 1.690 \\
\hline$R 32$ & $\mathrm{Gd}$ & $\mathrm{Cr}$ & 1.053 & 0.615 & 1.699 \\
\hline$R 32$ & $\mathrm{Sm}$ & $\mathrm{Fe}$ & 1.079 & 0.645 & 1.701 \\
\hline$R 32$ & $\mathrm{La}$ & $\mathrm{Sc}$ & 1.160 & 0.745 & 1.715 \\
\hline$R 32$ & $\mathrm{Gd}$ & $\mathrm{Fe}$ & 1.053 & 0.645 & 1.720 \\
\hline$R 32$ & $\mathrm{Ce}$ & $\mathrm{Sc}$ & 1.143 & 0.745 & 1.727 \\
\hline$R 32$ & $\mathrm{~Tb}$ & $\mathrm{Fe}$ & 1.040 & 0.645 & 1.729 \\
\hline$R 32$ & $\mathrm{Er}$ & $\mathrm{Cr}$ & 1.004 & 0.615 & 1.733 \\
\hline$R 32$ & $\mathrm{Er}$ & $\mathrm{Fe}$ & 1.004 & 0.645 & 1.754 \\
\hline$P 3{ }_{1} 21$ & $\mathrm{Y}_{0.94} \mathrm{Bi}_{0.06}$ & $\mathrm{Fe}$ & 1.028 & 0.645 & 1.737 \\
\hline$P 3_{1} 21$ & Dy & $\mathrm{Fe}$ & 1.027 & 0.645 & 1.738 \\
\hline$P 3_{1} 21$ & $\mathrm{Ho}_{0.963} \mathrm{Bi}_{0.037}$ & $\mathrm{Fe}$ & 1.021 & 0.645 & 1.742 \\
\hline$P 3_{1} 21$ & $\mathrm{Nd}$ & $\mathrm{Sc}$ & 1.109 & 0.745 & 1.751 \\
\hline
\end{tabular}

о всевозможных их структурах из баз данных COD (http://www.crystallography.net/cod/) и ICSD. Было найдено порядка 85 структур при разных температурах синтеза и съемки. Грубо все фазы можно классифицировать на тригональные: $R 32 ; P 3{ }_{1} 21 ; P 321$ и моноклинные: $C 2 / c$; $C c ; C 2$. Моноклинные фазы существенно отличаются от тригональных своей структурой и свойствами [13], и они в настоящей работе не рассматривались. Недавние исследования [14] показали, что фаза P321, наблюдаемая ранее в $\mathrm{NdSc}_{3}\left(\mathrm{BO}_{3}\right)_{4}$, является, скорее всего, ошибочной и для нее выбрана более корректная модель $P 3_{1} 21$. Именно поэтому наибольший интерес представили фазы $R 32$ и $P 3_{1} 21$, поскольку им принадлежит большинство депонированных структур. Кроме того, стоит отметить, что возможен фазовый переход $R 32 \leftrightarrow P 33_{1} 21$ как по температуре, так и по составу, что публиковалось ранее [15-18], поэтому вызывает наибольший интерес по выводу толеранс фактора именно для этих фаз. Используемые в настоящей работе тригональные фазы $R 32$ и $P 3_{1} 21$ (таблица), были исследованы при нормальных условиях. Температурные изменения симметрии мы в настоящей работе не рассматривали, поскольку толеранс-фактор подразумевает влияние лишь геометрических характеристик на симметрию, и нашей целью было выявление закономерностей лишь от соста- ва $R M_{3}\left(\mathrm{BO}_{3}\right)_{4}$. Стоит отметить, что зависимость температуры фазового перехода $R 32 \leftrightarrow P 3{ }_{1} 21$ от ионных радиусов уже ранее исследовалось [15] и сделан вывод о линейной зависимости $T=\mathrm{A} \cdot \mathrm{I} R(R)+\mathrm{B}$, где $\mathrm{A}, \mathrm{B}-$ коэффициенты, $\mathrm{I} R(R)$ - ионный радиус редкоземельного элемента (Y, La-Lu), что частично подтверждено в работе [16].

Для того чтобы установить закономерности преобразования $R 32$ в $P 3_{1} 21$ было выполнено тщательное сравнение структур $\mathrm{TbFe}_{3}\left(\mathrm{BO}_{3}\right)_{4}$ в фазах $R 32$ $(300 \mathrm{~K})$ и $P 3_{1} 21(2 \mathrm{~K})[18]$, при помощи программы ISODISTORT [19]. Обнаружено, что при фазовом переходе борный треугольник $\mathrm{B}^{\circ} \mathrm{O}_{3}$ и призма $\mathrm{TbO}_{6}$ не претерпевают особых изменений. Однако треугольник $\mathrm{B} 2 \mathrm{O}_{3}$ расщепляется на две позиции: $\mathrm{B}_{2} \mathrm{O}_{3}$ и $\mathrm{B} 3 \mathrm{O}_{3}$, и октаэдр $\mathrm{FeO}_{6}$ тоже расщепляется на две позиции: $\mathrm{Fe}_{6} \mathrm{O}_{6}$ и $\mathrm{Fe} 2 \mathrm{O}_{6}$. Согласно анализу мод искажений в ISODISTORT, наибольшее смещение в структуре испытывает атом O2, принадлежащий борному треугольнику $\mathrm{B}_{2} \mathrm{O}_{3}$ (рис. 2). Именно это критическое смещение приводит к изменению симметрии.

Атом О2 находится в полости, ограниченной атомами $\mathrm{Fe} 2-\mathrm{O} 2-\mathrm{B} 2-\mathrm{O} 7-\mathrm{Tb}-\mathrm{O}_{3}-\mathrm{B} 2-\mathrm{O} 7-\mathrm{Tb}-\mathrm{O} 4$ (рис. 2). Сделано предположение, что размер этой полости в направлении наибольшего смещения атома О2 (рис. 2) 


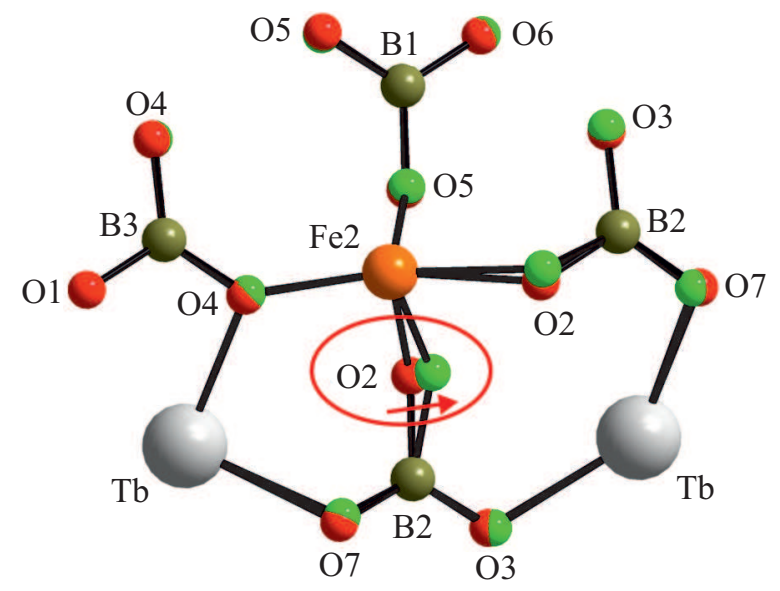

Рис. 2. Изменение структуры $\mathrm{TbFe}_{3}\left(\mathrm{BO}_{3}\right)_{4}$ при фазовом переходе из $R 32(300 \mathrm{~K})$ в $P 3{ }_{1} 21(2 \mathrm{~K})$. Кругом отмечен атом O2, испытывающий наибольшее смещение при фазовом переходе. Направление смещения при охлаждении показано стрелкой.

должен влиять на стабильность фазы $R 32$. Если полость небольшая, то атому $\mathrm{O} 2$ сложно перемещаться, в результате не происходит критического смещения и смены фазы в $P 3_{1} 21$, т. е. фаза остается $R 32$. Если же полость большая, то атом $\mathrm{O} 2$ легко смещается в направлении стрелки и фаза перестает быть $R 32$, и переходит в $P 3_{1} 21$.

Было решено вычислить размер этой полости исходя из информации об ионных радиусах ионов, составляю- щих структуру. Из рис. 3 видно, что эта полость равна разности между длиной верхнего ряда ионов

$$
\mathrm{L} 1=I R(\mathrm{~B})+2 \times I R(\mathrm{O})+2 \times I R(M)+I R(\mathrm{O})+I R(\mathrm{~B})
$$

и двумя ионными радиусами иона $R: 2 \times \mathrm{L} 2=2 \times \mathrm{I} R(R)$. Отношение разности $\mathrm{L} 1-2 \times \mathrm{L} 2$ к размеру иона кислорода $\mathrm{L} 3=2 \times I R(\mathrm{O})$ и является толеранс-фактором, определяющим симметрию фазы хантита для любого состава

$$
t=\frac{L 1-2 \times L 2}{L 3} .
$$

Подставив уравнение (1) в (2) и, сделав упрощения, получим

$$
t=\frac{I R(B+2 \times I R(\mathrm{O})+I R(M)-I R(R)}{I R(\mathrm{O})},
$$

где $\operatorname{IR}(\mathrm{O})=\operatorname{IR}\left(\mathrm{O}^{2-}, \mathrm{CN}=6\right)=1.42 \AA-$ ионный радиус иона кислорода, $I R(\mathrm{~B})=I R\left(\mathrm{~B}^{+3}, \mathrm{CN}=3\right)=0.01 \AA-$ ионный радиус иона бора, $I R(\mathrm{M})=I R\left(M^{3+}, \mathrm{CN}=6\right)$ $=0.6-0.75(\AA)-$ ионный радиус иона металла, $I R(\mathrm{R})=I R\left(\mathrm{Ln}^{3+}, \mathrm{CN}=8\right)=0.8-1.7(\AA)-$ ионный радиус иона редкоземельного элемента, $\mathrm{CN}$ - означает координационное число для конкретного иона. Для ионов редкоземельных элементов $R$ в настоящей работе используется координационное число $\mathrm{CN}=8$, вследствие того, что помимо 6 ближайших атомов кислорода с расстоянием $d(\mathrm{Ln}-\mathrm{O}) \sim 2.4 \AA$, существует еще два атома

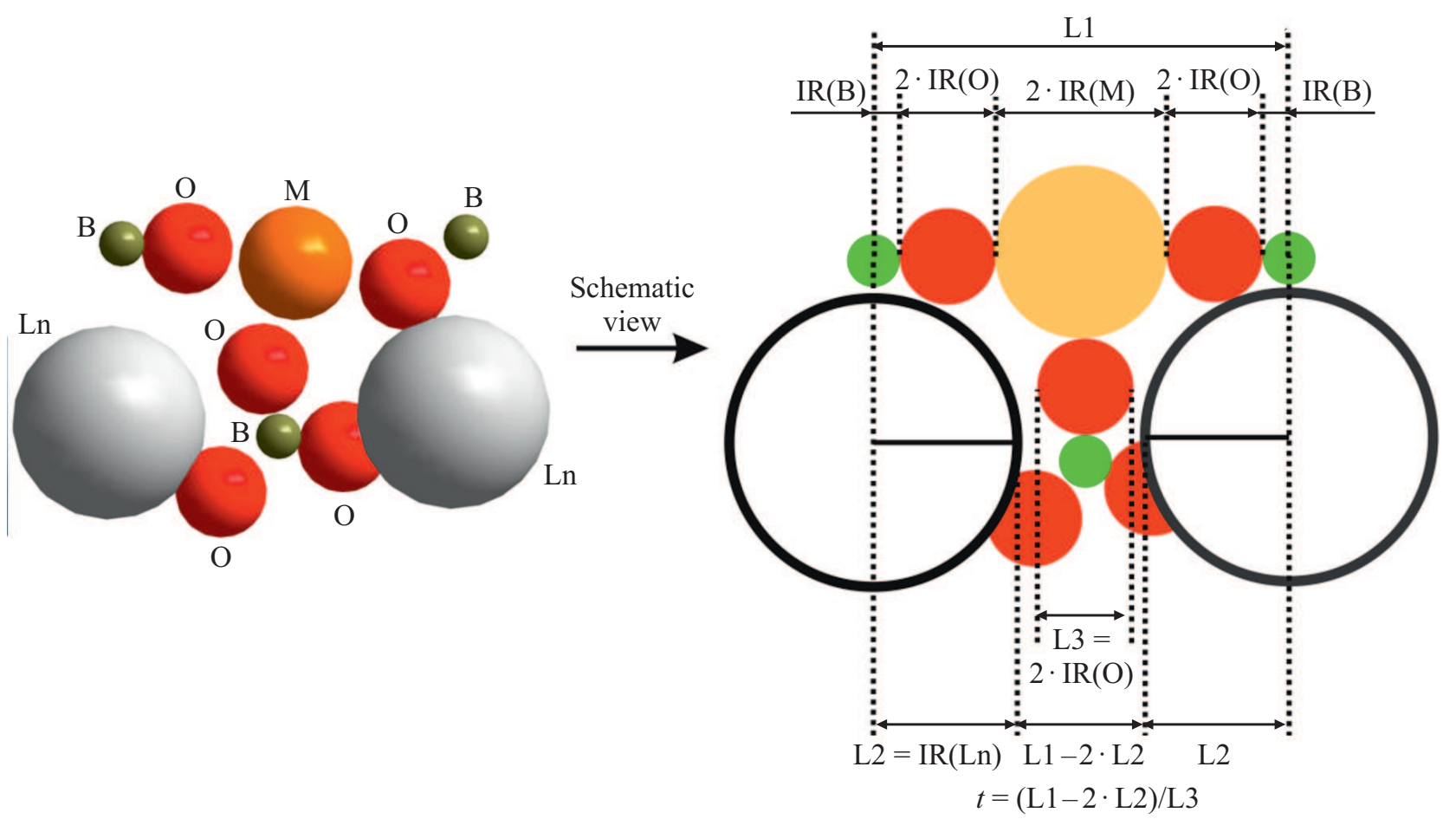

Рис. 3. Координационное окружение борного треугольника $\mathrm{BO}_{3}$ в $R M_{3}\left(\mathrm{BO}_{3}\right)_{4}-(a)$. Ионы $R^{3+}, M^{3+}, \mathrm{B}^{3+}, \mathrm{O}^{2}-$ представлены сферами с радиусами близкими к ионным радиусам. $(b)$ - характерные размеры ионов и основные длины, из которых впоследствии вычисляется толеранс-фактор $t$. 
кислорода с расстоянием $d(\mathrm{Ln}-\mathrm{O}) \sim 2.8 \AA$. В результате форма полиэдра является двухшапочной тригональной призмой. Значения ионных радиусов используются по Шеннону [20].

\section{3. Результаты}

Для всех исследуемых соединений была вычислен толеранс фактор по формуле (3) и вещества отсортированы в таблице по этому значению $t$, от минимального значения к максимальному. Такая сортировка разбила группы тригональных фаз на два подкласса, с пограничным значением толеранс фактора $t_{0}=1.737$. Значения факторов меньше этого числа, приводит к фазе $R 32$. Значения больше этого числа, приводит к фазе $P 3_{1} 21$, за исключением соединения $\mathrm{ErFe}_{3}\left(\mathrm{BO}_{3}\right)_{4}(R 32)$, которое имеет аномально большое значение фактора 1.754. Эта точка (рис. 4) может являться случайным выбросом, и, либо химический состав у этого кристалла другой, либо фаза на самом деле $P 3{ }_{1} 21$, а не $R 32$. Большинство же соединений очень хорошо разделилось на два подкласса, и можно считать, что формула толеранс фактора, действительно содержит информацию об области стабильности $R 32$-фазы.

Анализ формулы (3) вскрывает существующую разницу между вкладами ионов $M$ и $R$ в стабильность $R 32$-фазы $R M_{3}\left(\mathrm{BO}_{3}\right)_{4}$ соединений. Во-первых, увеличение ионного радиуса $R$ уменьшает толеранс фактор, и приводит к фазе $R 32$, а увеличение ионного радиуса $M$, наоборот, повышает вероятность реализации искаженной фазы $P 3_{1} 21$. Во-вторых, $I R(M)$ и $I R(R)$, имеют разные диапазоны значений: $I R(M)$ находится в пределах $0.6-0.75(\AA)$, а ионный радиус всех возможных $R$ ионов

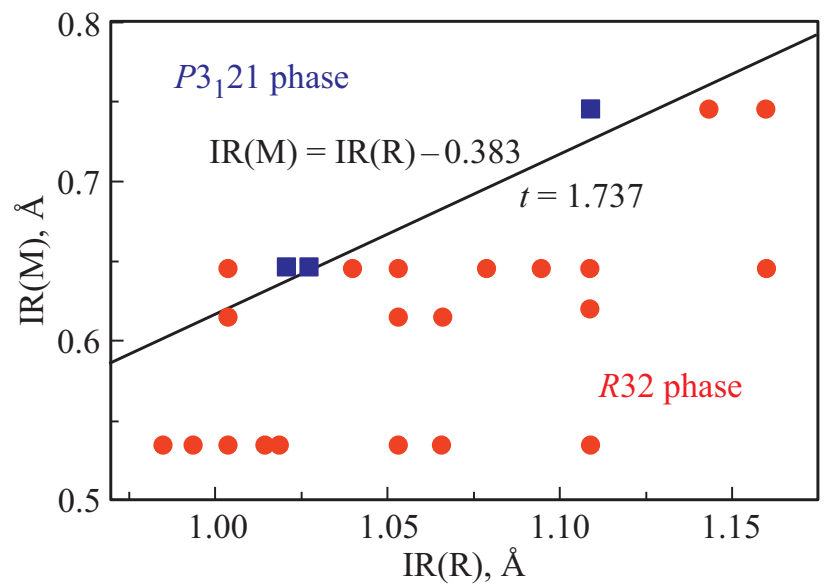

Pис. 4. Диаграмма распределения фаз $R 32$ (круги) и $P 3_{1} 21$ (квадраты) известных соединений $R M_{3}\left(\mathrm{BO}_{3}\right)_{4}$ по ионным радиусам $I R(M)$ и $I R(R)$. Наклонная линия характеризует пограничные соединения, у которых толеранс фактор равен

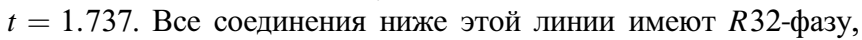
а выше $-P 3_{1} 21$. Только один круг находится не в своей зоне, что может являться выбросом. находится в пределах 0.8-1.25 (А) (таблица). Из этого следует то, что ион $R$ имеет больший вклад в толеранс фактор $t$, и большее влияние на стабильность фазы $R 32$.

Используя эти знания можно сразу предсказать, что соединения с $R=\mathrm{La}, \mathrm{Pr}, \mathrm{Nd}, \mathrm{Pm}, \mathrm{Sm}, \mathrm{Eu}$ будут, вероятнее всего, в R32-фазе, поскольку при любых известных $M$ толеранс фактор будет меньше значения 1.737. В этом легко убедиться рассчитав толеранс фактор для некоторых соединений, которые еще не были получены в реальных экспериментах. Например, рассмотрим соединения с химическим составом $\mathrm{PrFe}_{3}\left(\mathrm{BO}_{3}\right)_{4}$ и $\mathrm{LaAl}_{3}\left(\mathrm{BO}_{3}\right)_{4}$, и используем ионные радиусы $I R(\operatorname{Pr})=1.126 \AA, I R(\mathrm{Fe})=0.645 \AA \quad$ и $I R(\mathrm{La})=1.16 \AA, I R(\mathrm{Al})=0.535 \AA . \mathrm{B}$ результате получим $t\left(\mathrm{PrFe}_{3}\left(\mathrm{BO}_{3}\right)_{4}\right)=1.668$ и $t\left(\mathrm{LaAl}_{3}\left(\mathrm{BO}_{3}\right)_{4}\right)=1.567$, что гораздо меньше пограничного значения $t_{0}=1.737$ и скорее всего эти соединения будут существовать при нормальных условиях в фазе $R 32$. Стоит отметить, что для самого хантита $\mathrm{CaMg}_{3}\left(\mathrm{CO}_{3}\right)_{4}(\mathrm{IR}(\mathrm{Ca})=1.12 \AA$, $I R(\mathrm{Mg})=0.72 \AA, I R(\mathrm{C})=-0.08 \AA, I R(\mathrm{O})=1.42 \AA)$ толеран-фактор равен $t=1.662$, поэтому хантит должен существовать в фазе $R 32$, что на самом деле так. Поэтому формула вероятнее всего применима не только к боратам, но и другим соединениям с другим составом.

\section{4. Заключение}

В настоящий момент открыто большое количество хантитов $R M_{3}\left(\mathrm{BO}_{3}\right)_{4}$, но до сих пор не было четкого

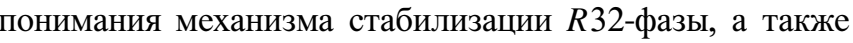
процессов, которые ведут к искажению структуры. В работе было установлено, что поворот лишь одного борного треугольника $\mathrm{BO}_{3}$ из двух, и как следствие, смещения одного атома кислорода, является наиболее критичным и приводит к фазовому переходу $R 32 \leftrightarrow P 3_{1} 21$. Понимание этого, позволило вывести формулу для толеранс фактора $t=\frac{I R(B+2 \times I R(\mathrm{O})+I R(M)-I R(R)}{I R(\mathrm{O})}$, что является значимым вкладом в дальнейшее изучение и предсказание структур класса хантитов.

Были рассчитаны значения толеранс фактора для уже существующих соединений класса хантита, и установлено, что значение $t_{0}=1.737$ является пограничным. Все толеранс-факторы, которые будут превышать данный порог значения, будут соответствовать фазе $P 3_{1} 21$.Все значения толеранс-фактора меньше 1.737 формируют область стабильности $R 32$-фазы.

Из формулы для толеранс фактора установлено, что чем больше значение ионного радиуса редкоземельного элемента $R$ и меньше значение ионного радиуса металла $M$, тем более вероятна фаза $R 32$. И наоборот: чем меньше ионный радиус $R$ и больше $M$, тем больше вероятность существования искаженной фазы $P 3_{1} 21$ при нормальных условиях.

Прогноз $t$-фактора хорошо описывает расслоение фаз соединений по симметриям, в чем можно убедиться из 
таблица и рис. 1. Поэтому можно с уверенностью применять данную формулу для прогноза новых соединений.

\section{Конфликт интересов}

Авторы заявляют, что у них нет конфликта интересов.

\section{Список литературы}

[1] D. Xue, K. Betzler, H. Hesse, D. Lammers. Solid State Commun. 114, 21 (2000).

[2] J.A. Campá, C. Cascales, E. Gutiérrez-Puebla, M.A. Monge, I. Rasines, C. Ruíz-Valero. Chem. Mater. 9, 237 (1997).

[3] K.-C. Liang, R.P. Chaudhury, B. Lorenz, Y.Y. Sun, L.N. Bezmaternykh, V.L. Temerov, C.W. Chu. Phys. Rev. B 83, 180417 (2011).

[4] J.-P. Meyn, T. Jensen, G. Huber. IEEE J. Quantum Electron.. 30, 913 (1994).

[5] I. Couwenberg, K. Binnemans, H. De Leebeeck, C. GörllerWalrand. J. Alloys Compd. 274, 157 (1998).

[6] D.A. Ikonnikov, A.V. Malakhovskii, A.L. Sukhachev, V.L. Temerov, A.S. Krylov, A.F. Bovina, A.S. Aleksandrovsky. Opt. Mater. 37, 257 (2014).

[7] H. Kronmüller, S. Parkin. General micromagnetic theory. Handbook of magnetism and advanced magnetic materials 1 (2007). 39 c.

[8] V.M. Goldschmidt. Naturwissenschaften 14, 477 (1926).

[9] X. Liu, R. Hong, C. Tian. J. Mater. Sci.: Mater. Electron. 20, 323 (2009).

[10] Z. Song, D. Zhou, Q. Liu. Acta Crystallographica C 75, 1353 (2019).

[11] R. Mouta, R.X. Silva, C.W.A. Paschoal. Acta Crystallographica B 69, 439 (2013).

[12] N.O. Azarapin, A.S. Aleksandrovsky, V.V. Atuchin, T.A. Gavrilova, A.S. Krylov, M.S. Molokeev, Sh. Mukherjee, A.S. Oreshonkov, O.V. Andreev. J. Alloys Comp. 832, 153134 (2020).

[13] A.S. Oreshonkov, E.M. Roginskii, N.P. Shestakov, I.A. Gudim, V.L. Temerov, I.V. Nemtsev, M.S. Molokeev, S.V. Adichtchev, A.M. Pugachev, Y.G. Denisenko. Materials 13, 545 (2020).

[14] E.V. Eremin, M.S. Pavlovskiy, I.A. Gudim, V. Temerov, M. Molokeev, N.D. Andryushin, E.V. Bogdanov. J. Alloys Comp. 828, 154355 (2020).

[15] Y. Hinatsu, Y. Doi, K. Ito, M. Wakeshima, A. Alemi. J. Solid State Chem. 172, 438 (2003).

[16] E. Moshkina, S. Krylova. I Gudim, M. Molokeev, V. Temerov, M. Pavlovskiy, A. Vtyurin, A. Krylov. Cryst. Growth Des. 20, 1058 (2020).

[17] M.S. Pavlovskii, N.D. Andryushin. Phys. Solid State 61, 2049 (2019).

[18] S.A. Klimin, A.B. Kuzmenko, M.A. Kashchenko, M.N. Popova. Phys. Rev. B 93, 054304 (2016).

[19] H.T. Stokes, D.M. Hatch, B.J. Campbell, D.E. Tanner. J. Appl. Crystallography 39, 607 (2006).

[20] R.D. Shannon. Acta cryst. A 32, 751 (1976).

Редактор Т.Н. Василевская 